\title{
MICROSCOPIC RADIATION QUANTITIES
}

\author{
Professor Harald H. ROSSI* \\ (manuscrit reģ le 2 mai 1968 )
}

\begin{abstract}
SUMMARY
The physical quantities ordinarily used in relation to ionizing radiation are macroscopic in that they represent averages over large numbers of particles and absorption events. However, many radiation effects and in particular cellular radiobiological effects are often due to the action of very few particles and frequently only due to one particle. Under these conditions the microscopic quantities which specify the energy concentrations actually existing can differ greatly from their macroscopic counterparts.

Among possible microscopic quantities the local energy density and the event size are defined and their inter-relation is discussed.

Brief reference is made to experimental determinations of event size distributions with walled and wall-less proportional counters.

A number of ways are considered in which microdosimetric quantities can be employed in theoretical radiobiology.
\end{abstract}

There is an inherent dualism between the theoretical and practical aspects of many physical quantities. As formulated in fundamental laws and evaluated in calculations these quantities are nearly always considered continuous in that they can assume any numerical value within their possible range of magnitudes or be differentiated with respect to length, time and other variables. On the other hand, the atomic nature of matter requires discontinuities even in such fundamental quantities as those of the MKSA System. Thus, for mass only certain values are possible for each element since any quantity of matter must have a mass that is very nearly ${ }^{1}$ an integral multiple of atomic or molecular weights. On the other hand, current which is the quotient of two quantities (charge and time) may have any value but must fluctuate about this because of the finite value of the electronic charge. Similar considerations apply to many other physical quantities.

Because of the large number of atomic units involved in most observable phenomena statistical uncertainties are usually of no importance. The grainy nature of both matter and radiation becomes important however when effects are due to only a few particles or quanta. In the simple case of the decay of a radioactive substance variations in the rate of disintegration are commonly observed and it is impossible to predict with any certainty the number of transformations

* Radiological Research Laboratory, Dept. of Radiology, Columbia University, New York.

1 The mass equivalent of chemical binding energies introduces very small modifications. 
that will occur in a sample during a period that is comparable to the reciprocal of its activity. This uncertainty is quite fundamental and it exists regardless of the accuracy to which the activity is known. One is obviously dealing with two entirely different quantities having equal dimensions $\left(\left[t^{-1}\right]\right)$ : the macroscopic quantity activity which is continuous and decreases steadily according to the decay constant; and the microscopic quantity represented by the quotient of the number of disintegrations divided by the time in which they occur. The first of these quantities is single valued and furnishes an expectation value for the second which is unpredictable and may assume a range of values distributed about a mean that is equal to the expectation value. The width of this distribution increases as the time period decreases. It it is much less than the reciprocal of the activity only values of $\circ$ and $\mathrm{I}$ occur with appreciable frequency. One might consider the macroscopic quantity to be more fundamental but one must admit that the microscopic quantity is more real since it is the actual rather than the expected number of disintegrations that must determine any effects.

In this example the relation between the activity and the probability distribution of the disintegrations in a given time period is well known since the former is the mean of the Poisson distribution constituted by the latter. The relation is much more complex in the case of another pair of associated quantities which also have the same dimensions. These are the absorbed dose, $D$, and the local energy density, z. Both of these refer to the quotient of $E / m$ where $E$ is the energy imparted by ionizing radiation to a mass $m$. However, $D$, the macroscopic quantity, is the average value of this ratio in a uniformly irradiated region while z, the microscopic quantity is the value actually obtaining in volumes of given shape and mass. $D$ is again the mean of the distribution $f(z)$ which represents the probability of the occurrence of $z$ per unit interval of $z$. The most important reason why the inactivation curves observed in cellular radiobiology are not step functions is that critical values of $z$ are reached only gradually as $D$ is increased. In the case of microorganisms a dose sufficient to kill a large fraction of the population can leave the survivors with local energy density of $\circ$.

As is the case with all quantities which are defined in terms of a quotient of two other physical quantities the discrepancies between the macroscopic and the microscopic concept becomes particularly large when the quantity in the denominator of the quotient becomes very small. In the case of absorbed dose and local energy density this denominator is $m$. In a medium which has been irradiated to receive some absorbed dose $D$ it is always possible to consider masses that are so small that it is unlikely that they have been traversed by any charged particle at all. The few instances when $z=0$ almost exclusively involve mass elements which have been traversed by only one particle. In these cases $z$ must be very high in order to obtain an average value $\bar{z}=D$ throughout the medium. Here $\bar{z}$ the average value of $z$ is defined by

$$
\bar{z}=\int_{0}^{\infty} z f(z) \mathrm{d} z .
$$

It is evident that the value of $m$ below which extreme fluctuations become important depends on the rate of energy deposition of the charged particles that deliver the absorbed dose. If this is large comparatively large values of $m$ will 
result in values of $z$ that are much larger than the absorbed dose. On the other hand if the rate of energy deposition is low $m$ must be small before fluctuations become important.

The macroscopic quantity commonly utilized to express the rate of energy deposition of charged particles is the linear energy transfer (LET). This is defined as $\Delta E / \Delta \mathrm{d}$ where $\Delta E$ is the energy locally deposited by a charged particle traversing a distance $\Delta \mathrm{d}$. The adjective "local » is uncertain and constitutes one of the weaknesses of the LET concept. The basic reason for the difficulty is that the tracks of charged particles are not line sources of deposited energy because of the production of secondary charged electrons (delta rays). Hence the description of energy deposition by a charged particle requires an additional macroscopic quantity. This might be the radial distribution of density of energy deposition.

The microscopic quantity which has the same dimensions as LET and is unaffected by such qualifications is the event size $y=E /$ d.* Here $E$ is the essentially instantaneous energy increment produced by the passage of a charged particle and/or any of its charged secondaries in a volume of stated shape and size; and $d$ is the average thickness of the volume, e.g. the average length of randomly oriented line segments traversing the volume. [3]

It can be shown that in a convex body the increment in local energy density, $z_{1}$, due to an event of size $y$ is given by [4]

$$
z_{1}=\frac{4 y}{s_{p}}
$$

where $\rho$ is the density of the medium and $S$ the body surface.

If $f(y)$, the probability distribution of event sizes, is known for the volume having the shape and size of interest one may derive $f(z)$ on the basis of the above formula for any value of the absorbed dose $D$. If the latter is sufficiently large a given value of $z$ will be due to several events. However from a knowledge of $f(y)$ one can determine the multiplicity of events at any dose, as well as the frequency per rad of the deposition of any amount of energy in excess of an arbitrary threshold, etc. [s] $f(y)$ is therefore the fundamental distribution for microdosimetry.

In the general case of a mass of tissue irradiated by indirectly ionizing radiation the spectrum $f(y)$ in a specified region depends on the following quantities:

I. The nature and energy distribution of the incident indirectly ionizing radiation.

2. The cross section for interactions whereby the indirectly ionizing radiation produces directly ionizing charged particles.

3. The nature and energy distribution of the resultant charged particles.

* The definitions given here for local energy density [I] and event size [2] are more general than those given previously when only spherical volumes were considered and $d$ was the sphere diameter. This change is indicated by the use of lower case rather than capital letters for the symbols of these quantities. In the case of local energy density the numerical values remain the same but in the case of event size the relation between the old and new quantity is given by $Y=2 / 3 y$. 
4. Complete information on the rate of energy loss of these particles including energy distributions in directions that are axial and radial relative to the track and including also the statistical fluctuations, the range energy relation and the degree of both straggling and track curvature.

All of this information is required in complete detail if one is to calculate the probability that a particle and/or associated delta rays deposit a given amount of energy in a given volume within irradiated matter.

Although extensive and sometimes even complete information is available on each of the above points, the remaining uncertainties and especially the mathematical complexity make a theoretical evaluation of $P(y)$ virtually impossible at present. On the other hand, the quantity may be reasonably accurately determined experimentally. In the only method employed thus far, this has been accomplished by a measurement of the ionization in tissue equivalent gas. The gas occupies a cavity of the required shape which is idential with the sensitive volume of a proportional counter. [6] An intrinsic limitations of this approach is that energy losses can only be determined in multiples of $W$, the average energy expended in the production of an ion pair. This minimum unit of about 30 electron volts is becoming the principal limit to the resolution of the device since it has become possible to readily detect single ionizations and to correct for the statistics of avalanche formation in the counter.

Until recently the sensitive volume of the proportional counter was surrounded by a wall of conducting tissue equivalent plastic. At the interface between gas and plastic there is a density variation by a factor of the ordre of 100,000 . The resultant backscattering of delta rays might introduce errors in the measurements and in addition a solid wall makes it impossible to perform measurements of the y spectrum of weakly penetrating charged particles such as natural alpha rays. Consequently, a new technique has been developed in which the sensitive volume is part of a much larger gas volume, the separation being accomplished only by electrical lines of force. [7]

$f(y)$ and $f(z)$ data published thus far have all been obtained with counters having solid walls. They include $f(y)$ spectra for neutrons ranging in energy from about $200 \mathrm{keV}$ to $\mathrm{I}_{4} \mathrm{MeV}$ [8] and preliminary data for Cobalt 60. [9]

Although it is evident that a numerical physical interpretation of radiobiological findings must require microdosimetry data, no systematic method for their application has as yet been developed. In the few instances in which a correlation has been attempted, three different approaches were chosen.

In the first and simplest of these a lower limit for the size of the radiation sensitive volume in a cell was derived on the basis of the obvious requirement that it must be large enough to contain on the average at least one charged particle at the average dose required to kill the cell. The result obtained was of some interest since it turned out that in the case of the very sensitive cells involved (the spermatogonia of the mouse), the sensitive volume is larger than the nuclear volume. [10]

In view of differences in the biological effectiveness between different radiations and the usual finding that this increases as the LET of the particles increases, one may go a step further and postulate that biological effects do not merely require the expenditure of energy in sensitive regions of the cell but that there is also a threshold level of energy concentration (or event size in the case of inactivation 
by single particles) below which the biological effect is not evoked. Differences in the RBE of fast neutrons in the induction of a chromosome break in maize could be accounted for on the assumption of a threshold event size in a volume which is nearly equal to the fraction of the nuclear volume which the chromosome segment should occupy during interphase. The model accounted not only for relative but also for absolute break frequencies. [i $\mathrm{I}]$

A third and perhaps more general application of microdosimetric concepts was developed when it was found impossible to account for the RBE of $0.43 \mathrm{MeV}$ neutrons relative to is $\mathrm{MeV}$ neutrons on the basis of any threshold energy density in spherical sites of any diameter. [12] It was also found necessary to exclude the assumption that several particles would be required to provide the threshold energy or that statistically independent energy deposition in several targets is involved. The only model which was found to be compatible with physical reality proved to be identical to a mechanism proposed by Neary in connection with chromosome breaks in plants. [13] According to this interpretation, the inactivation occurs as a result of energy deposition in two loci having a definite geometrical relation with respect to each other. Inactivation occurs not only by energy deposition in each site by separate particles but also by the virtually instantaneous deposition of energy in both sites by one high LET particle. In the latter case the inactivation events are statistically interdependent.

This hypothesis is equivalent to the assumption that the target implicated in radiation injury has a structure and that microdosimetric measurements need to be concerned with coincidences in multiple volumes. Indeed in the common case of higher biological effectiveness of high LET relative to low LET radiation, an energy concentration must be involved in which several absorption events occur in sites that are in close proximity. Unless these sites are randomly distributed within some volume, a single sensitive volume cannot be said to exist and the only realistic interpretation requires that one consider each of the constituent sites in their relative geometrical relation.

It is apparent that the large number of parameters such as size and shape of sensitive volume, energy threshold, multiplicity, and geometrical inter-relation makes it impossible to systematically consider all possible combinations for even one radiation. It would be even more impractical to attempt to fit such physical data to each of the various inactivation models which have been proposed in theoretical radiobiology. The usefulness of microdosimetric quantities has been and very likely will be that they can be employed to permit the simplest theories of the action of radiation that are consistent with physical facts. The development of effective methods for the measurement of the pertinent microscopic quantities involved will continue to present a challenge to the radiological physicist.

\section{REFERENCES}

[I] Rossi, H.H., Binvati, M.H., and Gross, W. Local energy density in irradiated tissues. I. Radiobiological significance. Radiation Researcb I5, No. 4, 431-439, 196 I.

[2] Rossi, H.H. Specification of radiation quality. Radiation Researcb 10, No. 5, 522-531, 1959.

[3] Bevan, J.S. Parameters describing the microscopic distribution of radiation dose. Pbysics in Med. and Biol. 11 , No. 3, 405-409, 1966. 
[4] Rossi, H.H. Microscopic distribution of radiation energy. Presented at Symposium on Microdosimetry sponsored by Euratom Ispra, Italy, Nov. 1967.

[s] Rossi, H.H. Energy distribution in the absorption of radiation. Chapter in Advances in biological and medical physics. Academic Press, 1968.

[6] Rossi, H.H. Microscopic energy distribution in irradiated matter. Chapter in end edition of " Radiation dosimetry. " Academic Press, 1968.

[7] Binvati, B.J., Gross, W., Rossi, H.H., and Kellerer, A.M. Annual report on research project. U.S. Atomic Energy Commission, Document NYO-2740-5, 6I-68, Jan. I, I968.

[8] Binvati, M.H., Rosenzweig, W., Rossi, H.H., and Mryanaga, I. The dependence of RBE on the energy of fast neutrons. III. Evaluations of radiation quality. Radiation Research I0, No. 3, 512-525, 1963 .

[9] Binvati, M.H. and Boer, E. Annual report on research project. U.S. Atomic Energy Commission, Document No. NYO-2740-3, 67-80, Jan. I, 1966.

[10] Rossi, H.H. Distribution of radiation energy in the cell. Radiology 78 , No. 4, 530-535, 1962.

[ir] SMrri, H.H. and Rossi, H.H. Energy requirements and RBE for producing a cytogenetic. phenomenon in maize by irradiating seeds with X-rays and monoenergetic neutrons. Radiation Research 28, No. 2, 302-321, 1966.

[12] Rossi, H.H. The role of associated absorption events in lenticular radiation injury. To be published in Proceedings of Second Panel on Biophysical Aspects of Radiation Quality, IAE $A$, Vienna, Apr. 1967 .

[13] Neary, G.J. Chromosome aberrations and the theory of RBE. I. General considerations. International J. Radiation Biol. 9, No. 5, 477-502, 1965. 